\title{
Synthetic cannabinoid-related ED visit trends in Maryland using ESSENCE, 2013-2018
}

\section{Adejare Atanda, Jessica C. Goodell, Sherry Adams, Veronica Black}

Maryland Deartment of Health, United States

Objective

Develop a free text query to track synthetic cannabinoid-related ED visits. Assess trends in synthetic cannabinoid use from 2013-2018 using spatial and time-series analysis.

\section{Introduction}

Maryland utilizes ESSENCE for identification of emerging public health threats, including non-fatal overdoses. Synthetic cannabinoids are heterogeneous psychoactive compounds identified as substances of abuse [1]. In March 2018, the Illinois Department of Public Health received reports of unexplained bleeding in patients who reported using these products [2]. As a result, CDC initiated coordination of national surveillance activities for possible cases of coagulopathy associated with synthetic cannabinoids use. By May 2018, state health departments reported 202 cases, including five deaths [3].

On April 3, 2018, Maryland reported its index case - a female in her 20's who presented to an ED with nausea, blood in her stool, vaginal bleeding, bruising, an elevated internal normalized ratio (>12.2), and bleeding oral ulcers after quitting use of a synthetic cannabinoid. She was successfully treated with Vitamin K. The first reported mortality in a Maryland resident was a male in his 30's who called EMS for fever and blood in his urine but subsequently went into cardiac arrest and was unable to be resuscitated. The patient was known to use synthetic cannabinoids. Brodifacoum exposure was confirmed by laboratory testing. As of September 2018, the Maryland Poison Control Center had received reports of 43 cases, and 3 deaths linked to the outbreak.

\section{Methods}

To support surveillance and timeliness of synthetic cannabinoids reporting, we developed a case definition by conducting key word searches to identify terms/phrases used by providers in Maryland ED's to document synthetic cannabinoid visits. This process yielded the following terms: "synthetic marijuana", "spice", and "K2".

Subsequently, we created a free text query based on the case definition and variations of the terms/phrases. This query allowed us to capture data on ED visits for synthetic cannabinoid use in the chief complaint (CC), discharge diagnosis (DD), and clinical impression (CI) fields of ESSENCE data.

Finally, descriptive and geographic spatial analyses were conducted of synthetic cannabinoid-related morbidity (ED visits) for 2013-2017 (data for 2018 is incomplete); and time trends analyzed for 2013-2018.

\section{Results}

From 2013 to 2017, a total of 1,097 ED visits across Maryland were synthetic cannabinoid-related (Table 1). The overall crude synthetic cannabinoid-related ED visit rate was 20 per 100,000 population. The number of synthetic cannabinoid-related ED visits increased 8-fold, from 40 in 2013 to 353 in 2017. Females made the most synthetic cannabinoid-related ED visits ( $\mathrm{n}=861$, $78 \%$ ). Adults aged 15-24 and 25-34 made $349(32 \%)$ and $367(33 \%)$ visits respectively to an ED for a synthetic cannabinoidrelated event. Whites and blacks made $466(42 \%)$ and $498(45 \%)$ visits respectively to an ED for a synthetic cannabinoid-related event. People who were non-Hispanic $(\mathrm{n}=988,90 \%)$, black $(\mathrm{n}=498,45 \%)$, female $(\mathrm{n}=861,78 \%)$, and aged $25-34(367,33 \%)$ visited an ED for a synthetic cannabinoid-related event more than any other demographic group.

Time trend analysis shows an increase from baseline in synthetic cannabinoid-related ED visits starting from July 2014 (Figure 1). Three spikes are noted thereafter in April, July, and September 2015 respectively. Consequently, ED visits for synthetic 
cannabinoid-related events dropped to a new baseline value in December 2015. Two spikes are also noted for synthetic cannabinoid-related ED visits in May and September 2017 respectively with a new baseline established starting January 2018.

Spatial analysis shows geographic clustering of synthetic cannabinoid-related morbidity in three Maryland jurisdictions; Baltimore City, Fredrick County, and Washington County (Figure 2).

The top five Maryland counties with crude synthetic cannabinoid-related ED visit rates included Allegany, Baltimore City, Frederick, St. Mary's and Washington; ranging from 87 in Washington county to 38 in St. Mary's county. The top ten crude synthetic cannabinoid-related ED visit rates per 100,000 population from 2013 to 2017 among all Maryland ZIP codes ranged from 87 in Washington county to 38 in St. Mary's county.

Spatial analysis also shows that hospitals with the greatest burden of synthetic cannabinoid-related ED visits were close to ZIP codes of communities with high crude synthetic cannabinoid-related ED visit rates (Figure 3).

\section{Conclusions}

Data from the ESSENCE program can be considered acceptable for monitoring synthetic cannabinoid-related ED visits in Maryland. It is useful for obtaining near real-time data about synthetic cannabinoid-related events, and as we have shown in our analysis, for the identification of key groups and geographic locations most in need of targeted interventions to reduce morbidity and mortality. Finally, it also provides us with the ability to retrospectively identify outbreaks, and to link data trends to ongoing interventions.

\section{References}

1. Riederer AM, et al. 2016. Acute Poisonings from Synthetic Cannabinoids - 50 U.S. Toxicology Investigators Consortium Registry Sites, 2010-2015. MMWR Morb Mortal Wkly Rep. 65, 692-95. PubMed https://doi.org/10.15585/mmwr.mm6527a2

2. Horth, Roberta. Notes from the Field: Outbreak of Severe Illness Linked to the Vitamin K Antagonist Brodifacoum and Use of Synthetic Cannabinoids - Illinois, March-April 2018.

3. Centers for Disease Control and Prevention. Outbreak of life-threatening coagulopathy associated with synthetic cannabinoids use. May 2018. Retrieved from: https://emergency.cdc.gov/han/han00410.asp

Table 1. Synthetic cannabinoid-related morbidity (ED visits), by year and demographic characteristics, 2013-2017 
Demographic

Characteristics

\begin{tabular}{|c|c|c|c|c|c|c|}
\hline & 2013 & 2014 & 2015 & 2016 & 2017 & $\begin{array}{l}\text { Grand } \\
\text { Total }\end{array}$ \\
\hline $\begin{array}{l}\text { Total synthetic } \\
\text { cannabinoid-related } \\
\text { ED visits }\end{array}$ & $40(4)$ & $68(6)$ & $420(38)$ & $216(20)$ & $353(32)$ & 1097 \\
\hline \multicolumn{7}{|l|}{ Sex } \\
\hline Male & $13(1)$ & $20(2)$ & $90(8)$ & $48(4)$ & $65(6)$ & 236 \\
\hline Female & $27(2)$ & $48(4)$ & $330(30)$ & $168(15)$ & $288(26)$ & 861 \\
\hline \multicolumn{7}{|l|}{ Age, years } \\
\hline $11-14$ & $4(<1)$ & $5(<1)$ & $5(<1)$ & $2(<1)$ & $2(<1)$ & 18 \\
\hline $15-24$ & $21(2)$ & $32(3)$ & $144(13)$ & $69(6)$ & $83(8)$ & 349 \\
\hline $25-34$ & $8(1)$ & $16(1)$ & $157(14)$ & $64(6)$ & $122(11)$ & 367 \\
\hline $35-44$ & $6(1)$ & $8(1)$ & $47(4)$ & $37(3)$ & $72(7)$ & 170 \\
\hline $45-54$ & $1(<1)$ & $4(<1)$ & $42(4)$ & $26(2)$ & $42(4)$ & 115 \\
\hline $55-64$ & (0) & $2(<1)$ & $22(2)$ & $15(1)$ & $26(2)$ & 65 \\
\hline $65-74$ & (0) & (0) & $3(<1)$ & $2(<1)$ & $5(<1)$ & 10 \\
\hline $75-84$ & (0) & $1(<1)$ & (0) & (0) & $1(<1)$ & 2 \\
\hline $85+$ & (0) & (0) & (0) & $1(<1)$ & (0) & 1 \\
\hline \multicolumn{7}{|l|}{ Race } \\
\hline White & (0) & $33(3)$ & $179(16)$ & $98(9)$ & $156(14)$ & 466 \\
\hline Black & (0) & $20(2)$ & $191(17)$ & $106(10)$ & $181(16)$ & 498 \\
\hline Asian & (0) & (0) & $3(<1)$ & (0) & $2(<1)$ & 5 \\
\hline Other & (0) & $3(<1)$ & $13(1)$ & $10(1)$ & $14(1)$ & 40 \\
\hline Unknown & $40(4)$ & $12(1)$ & $34(3)$ & $2(<1)$ & (0) & 88 \\
\hline \multicolumn{7}{|l|}{ Ethnicity } \\
\hline Hispanic & (0) & $4(0)$ & $5(0)$ & $6(1)$ & $5(<1)$ & 20 \\
\hline Non-Hispanic & (0) & $51(5)$ & $381(35)$ & $208(19)$ & $348(32)$ & 988 \\
\hline Unknown & $40(4)$ & $13(1)$ & $34(3)$ & $2(<1)$ & $(0)$ & 89 \\
\hline
\end{tabular}

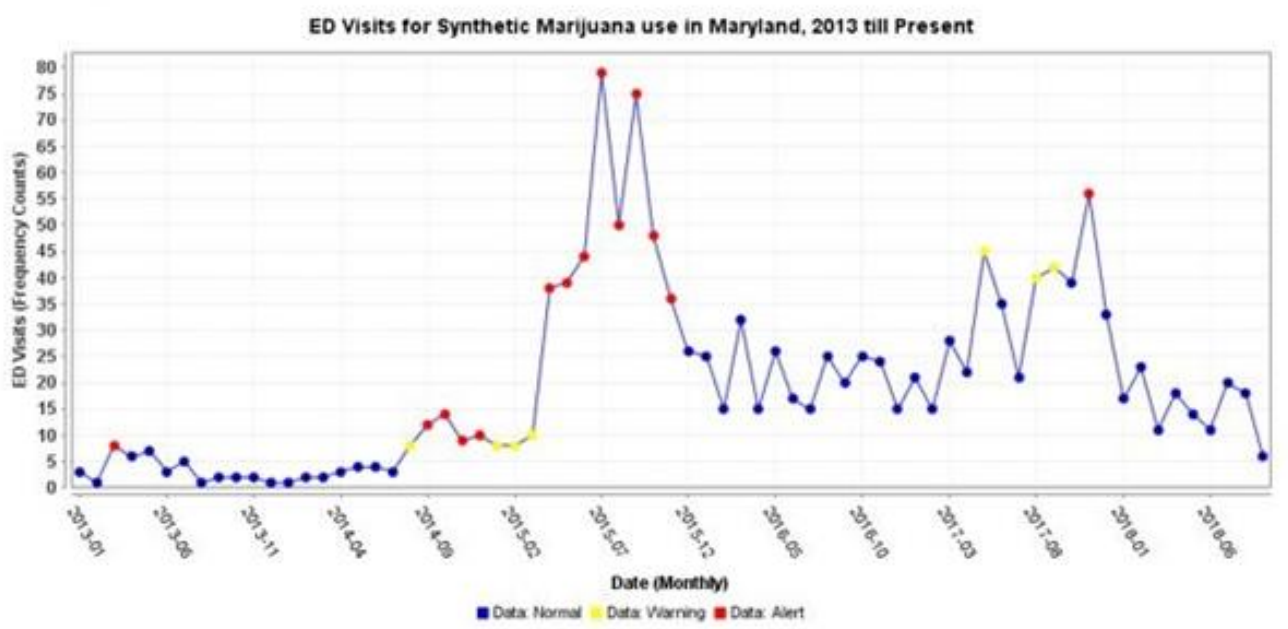

Figure 1. Monthly time trends of synthetic cannabinoid-related ED visits, Maryland, 2013-2018 


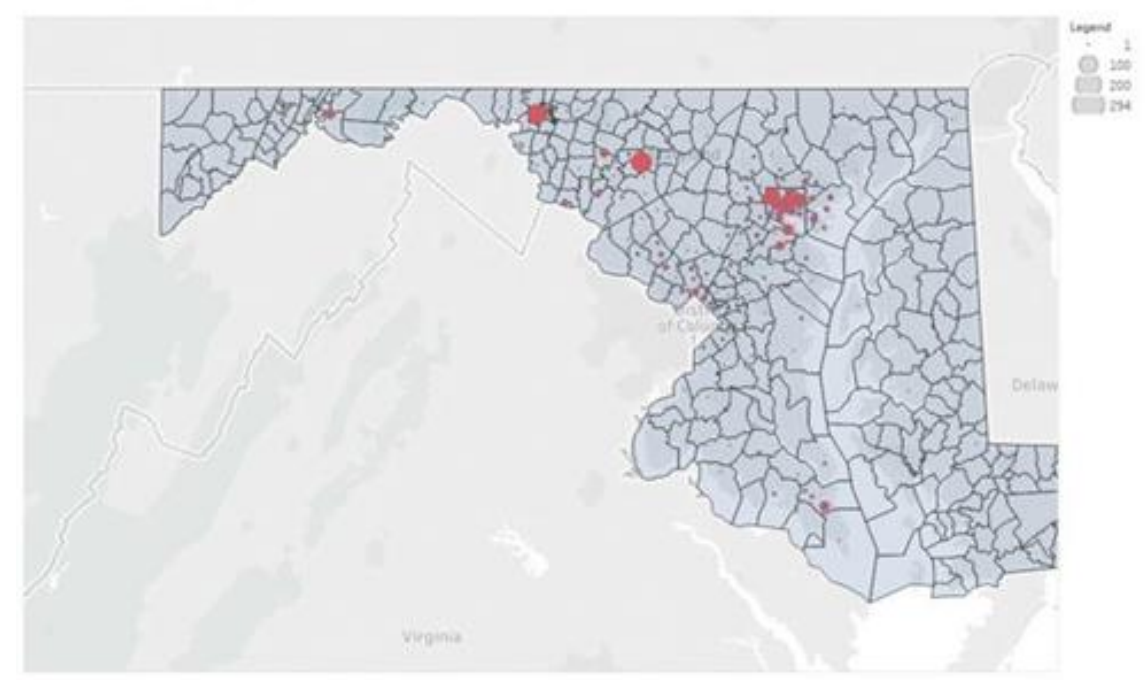

Figure 2. Geographic spatial analysis showing clustering of synthetic cannabinoid-related ED visits by patient residence, Maryland, 2013-2017.

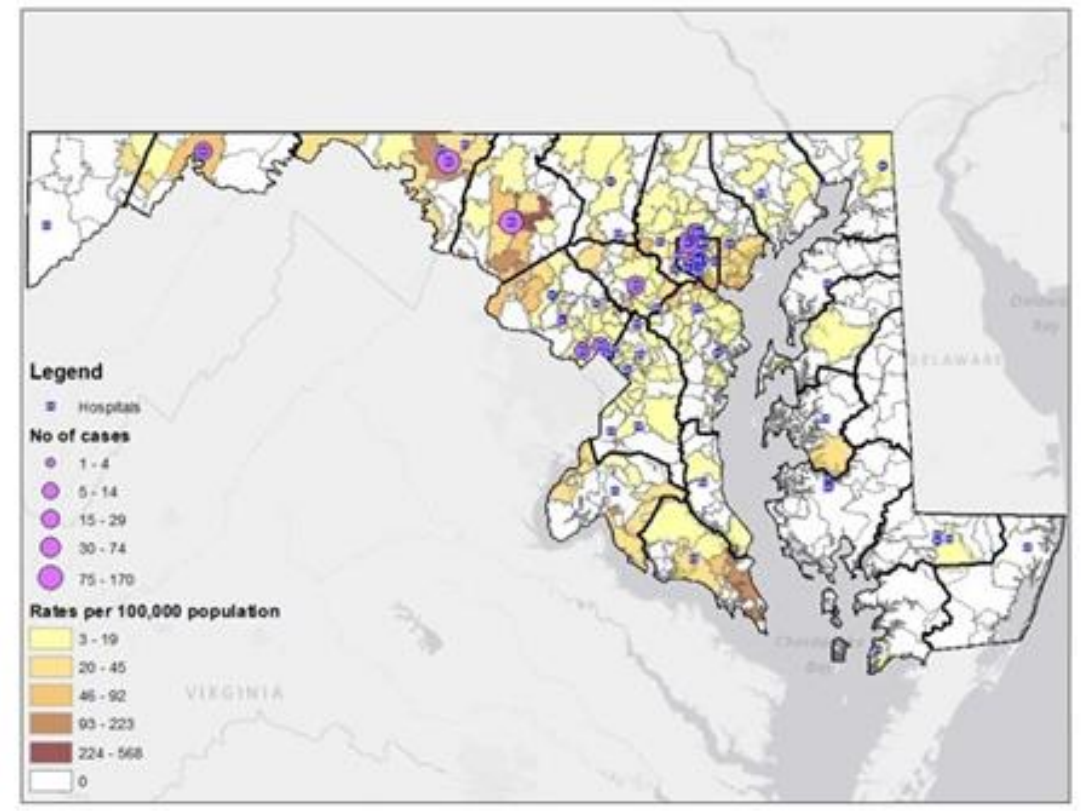

Figure 3. Geographic spatial analysis by ZIP code (patients) and total number of synthetic cannabinoid-related ED visits by hospital, Maryland, 2013-2017. 